\title{
Ein jambisches Gedicht des Andreas von Kreta.
}

I.

In allgemeinen Umrissen ist uns das Leben des Erzbischofs Andreas von Kreta bekannt. ${ }^{1}$ ) In Damaskus geboren, kam er in seinem 15. Lebensjahre nach Jerusalem, wurde Mönch und trat bald in nahe Beziehungen zu den Patriarchen der heiligen Stadt. Sophronios, der bekannte Homiletiker und Verfasser des Lebens der Maria Aegyptiaca ${ }^{2}$ ), soll dies nach dem Synaxarion gewesen sein, das uns in dem „grolsen Kanon" des Andreas überliefert wird. ${ }^{3}$ ) Die Unrichtigkeit dieser Angabe, die übrigen schon Papebroch bezweifelt hat Acta SS. I 69, wird dadurch erwiesen, dafs Sophronios im Jahre 638 starb $^{4}$ ), Andreas aber sicher noch im Jahre 713 lebte (s. u. S. 513 ff.), wahrscheinlich auch noch beim Ausbruch des Bilderstreites unter Leo dem Isaurer schriftstellerisch thätig war (726)..$^{5}$ ) Auch die anderen Nachrichten des Synaxarion kommen mir zweifelhaft vor. Des Sophronios Vita der ägyptischen Maria und seinen eigenen ,grofsen Kanon" soll Andreas nach Konstantinopel gebracht haben, als er von Theodoros von Jeru-

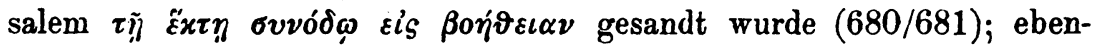
dort habe er energisch gegen den Monotheletismus gestritten. ${ }^{6}$ ) Ehrhard behauptet deshalb (a. a. 0.), Andreas sei als Vertreter des Patriarchen

1) Vgl. A. Ehrhard bei K. Krumbacher, Byz. L. ${ }^{2} 165$ f., wo auch die ältere Litteratur verzeichnet ist aufser W. Christ, Anthol. graec. carm. christ. XLII.

2) Vgl. A. Ehrhardt a. a. O. S. 188 f., K. Krumbacher ebd. S. 672 f.

3) Das Synaxarion findet sich auch, was Ehrhard a. a. O. übersehen hat, bei Migne, Patr. gr. 97 col. 1361-1364.

4) Ehrhard a. a. 0 .

5) Wenigstens ist bis jetzt kein Grund vorhanden, ihm das im Cod. Paris. 1630 unter seinem Namen überlieferte Fragment über die Verehrung der Bilder abzusprechen.

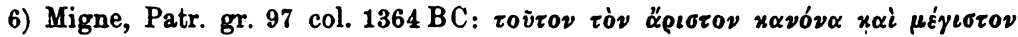

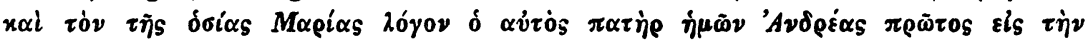

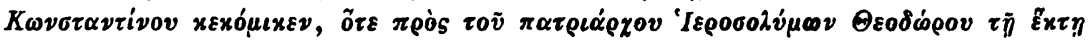

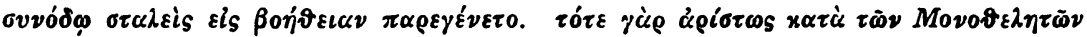
$\dot{\alpha} \boldsymbol{\omega} \omega \nu \iota \sigma \dot{\alpha} \mu \varepsilon \nu 0 s \% \tau \lambda$. 


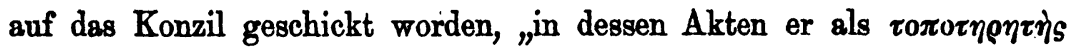

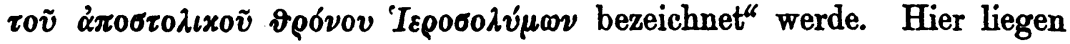
verschiedene Irrtümer zu Grunde. In den Akten des 6. Konzils (Harduin III col. 1043-1645) habe ich den Andreas nicht ein einziges Mal. auch nur genannt gefunden, dagegen wird in der Präsenzliste aller 18 Sitzungen jedesmal ein Georgios als Vertreter des Stuhles von Jerusalem genannt mit der stets wiederkehrenden Formel: $\Gamma \varepsilon \omega \rho y i o v$

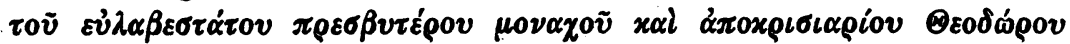

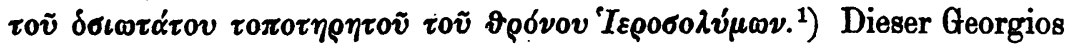
hat in der That $\mathrm{zu}$ wiederholten Malen in bedeutender Weise in den

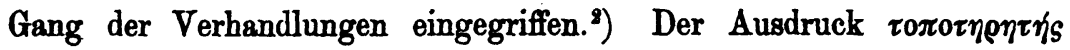
bedeutet aber auch gar nicht "Vertreter", sondern es ist, wie aus der Formel deutlich hervorgeht, damit Theodoros selbst bezeichnet; dieser war eben, wie ihn Hefele richtig genannt hat (a. a. 0. S. 274), nicht Patriarch von Jerusalem, sondern Patriarchalvikar. Indessen kann Andreas immerhin diesen Apokrisiar Georgios nach Konstantinopel begleitet haben, und besonders haben wir nicht den geringsten Grund, daran zu zweifeln, dafs er damals ein mehr oder weniger überzeugter Anhänger der Orthodoxie war. Auch die weitere Bemerkung im

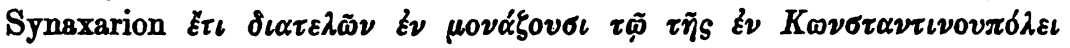

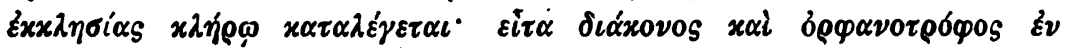

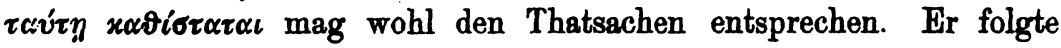
dann dem Beispiel eben jenes Apokrisiars Georgios, der ebenfalls während seiner Thätigkeit an den Verhandlungen des Konzils in den Klerus der Hauptstadt übertrat. ${ }^{3}$ )

Aus seinem ferneren Leben ist wenig bekannt. Er wurde zum Erzbischof von Kreta ernannt und wulste sich gleich manchen anderen

1) In den lateinischen Akten der 11. Sitzung (Harduin a. a; 0. col. 1254) steht Petro Deo amabili presbytero monacho et apocrisiario Theodori venerabilis loci servatoris sedis Hierosolymorum. Allein hier ist eine Liicke, vielleicht nur ein Druckfehler, denn im griechischen Text lesen wir hier wie immer: İ́rpov

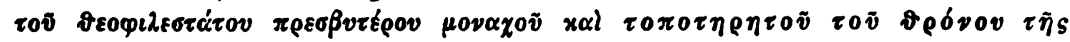

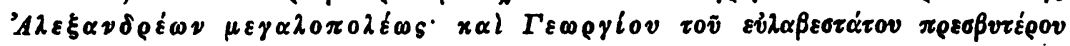

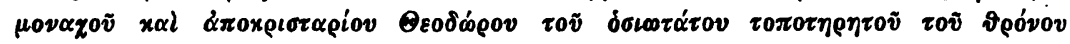
'IEporodónuv. III $274 \mathrm{ff}$.

2) Vergleiche das Nahere hierüber bei v. Hefele, Konziliengesch. 2. Aufl.

3) In den Unterschriften unter den Konzilsakten lesen wir (Harduin 1. c.

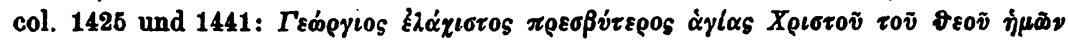

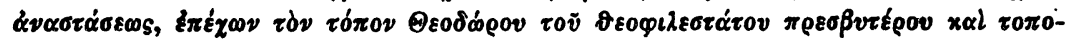

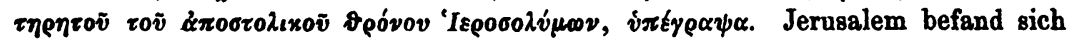
damals in den Händen der Araber. 
Kirchenfürsten den Wünschen der Regierung anzupassen, als für kurze Zeit mit Philippikos Bardanes (711-713) der Monotheletismus wieder zur Herrschaft kam. Nachdem aber am Pfingstsonntage 713 Bardanes geblendet und Anastasios II auf den Thron erhoben war, wurde die Orthodoxie wieder feierlich in ihre Rechte eingesetzt und schien inzwischen keipe Anhänger verloren zu haben; mit dem Patriarchen Johannes bekannte sich auch der Erzbischof Andreas von Kreta wieder zur reinen Liehre. ${ }^{1}$ )

I.

Der urkundliche Beweis für diesen letzten Glaubenswechsel des Andreas ist uns in einem Gedichte erhalten, das um so mehr Beachtung verdient, als es das einzige in Jamben verfafste Werk des Erzbischofs ist, dessen Namen wir sonst unter den Dichtern des Kirchenliedes zu nennen pflegen. Es ist an den Archidiakon und Chartophylax Agathon in Konstantinopel gerichtet und nicht, wie Krumbacher glaubte (Byz. Litt. ${ }^{2}$ 674), unediert, sondern öfter gedruckt worden. Nach einem Cod. Tilianus ${ }^{2}$ ) wurde das Gedicht zuerst von Combefisius mit einer lateinischen Übersetzung herausgegeben in seiner Historia haeresis Monothelitarum, Parisiis MDCXLVIII col. 235-240; es wurde abgedruckt in der Bibliotheca veterum patrum Andreae Gallandii, Venetiis MDCCLXXIX p. 167-169, der eine neue lateinische Übersetzung versuchte, und diese Ausgabe wurde mit der Übersetzung wiederholt bei Migne, Patr. gr. 97 col. 1437-1444.

Im Folgenden gebe ich den Text des Gedichtes nach der Ausgabe von Combefis (C) und den. Codd. Ambros. gr. J 91 inf. saec. XVI fol. $102^{\mathrm{r}}-104^{\mathrm{r}}$ (A) und Monac gr. 198 saec. XVI fol. $249^{\mathrm{r}}-250^{\mathrm{r}}$ (M). Das Gedicht ist noch in anderen Hss überliefert, von denen Krumbacher a. a. 0 . den Cod. Vatic. Reg. Suec. 55 fol. 260 -261 anführt; doch genügen zur Feststellung des Textes im ganzen die eben genannten Hilfsmittel. Eine neue Ausgabe ist um so notwendiger, als es geradezu unmöglich ist, die Verse nach den bisherigen Drucken zu verstehen. In Combefis' Vorlage standen Vers 1, 3, 5 u. s. w. links, Vers 2, 4, 6 u. s. w. auf der rechten Hälfte derselben Seite. Anstatt nun aber von links nach rechts zu lesen, las er zuerst die linke Hälfte der Seite von oben nach unten und druckte Vers $1,3,5$ u. s. w. bis 45 , danm V. 2, 4, 6 u. s. w. bis 46 u. s. w.; quandoque dormitat Homerus.

1) Die Annahme von Oudinus, Supplementum de scriptt. eccles. p. 190, da「s Andreas zuletzt Erzbischof von Kaisarea in Kappadokien geworden sei, ist falsch.

2) Aus der Bibliothek des Johannes du Tillet Baron de la Buissière. Vgl. Combefis. Vorrede candido lectori. In der Nationalbibliothek von Paris befindet sich die $\mathrm{Hs}$ nicht. 
Merkwürdig ist daher seine Ḿeinung (in den notae col. 240) clara satis mente Andreae ac vena facili, und die lateinische Übersetzung mulste ihm nicht ganz leicht werden. Sie befriedigte denn auch Gallandius so wenig, dafs er eine eigene neue an ihre Stelle setzte; um den griechischen Text hat dieser Gelehrte sich wohl gar nicht bekümmert, und über den Nachdruck bei Migne brauche ich nichts zu sagen.

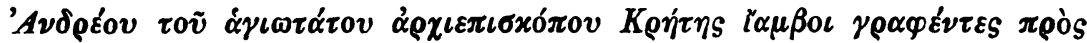

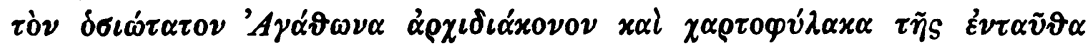

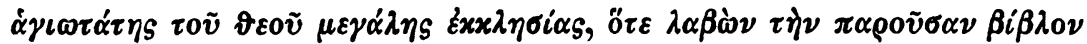

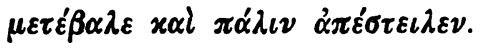

'E

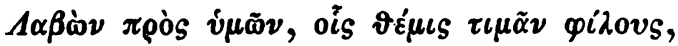

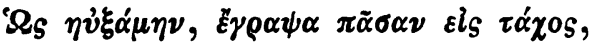

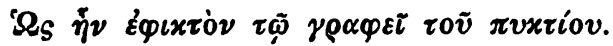

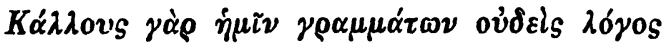

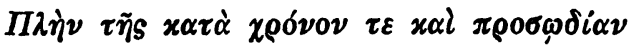

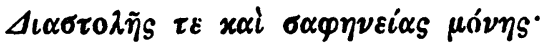

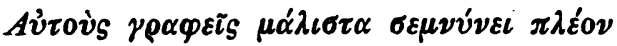

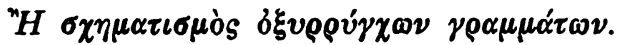

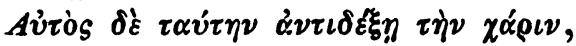

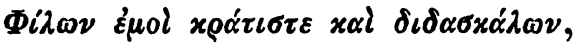

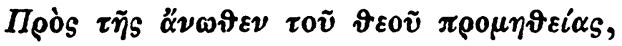

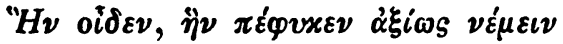

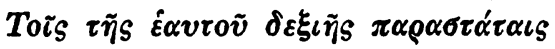

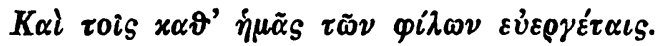

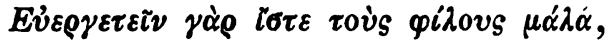

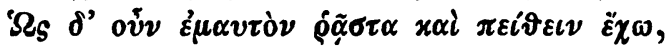

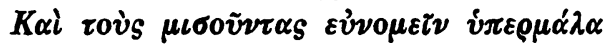

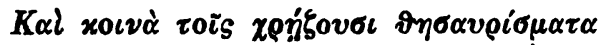

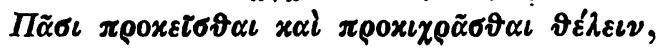

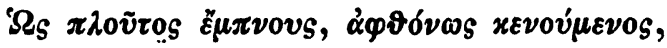

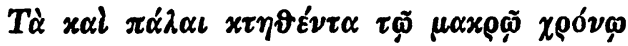

Cod. Ambr. J 91 inf. = A, Cod. Monac. $=$ M, ed. Combefis. = C. tit.: post

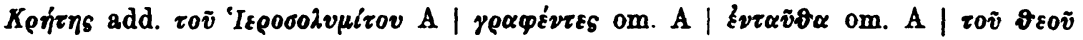

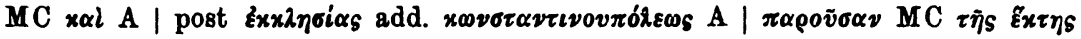

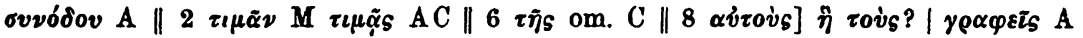

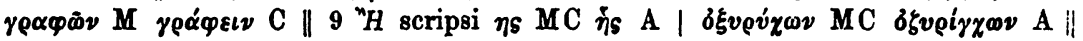

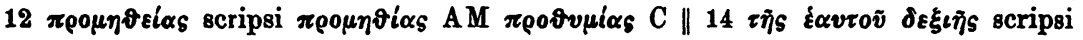

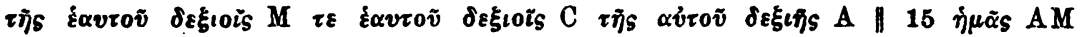

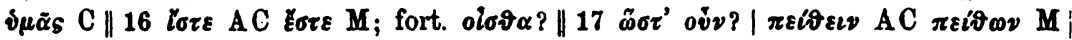

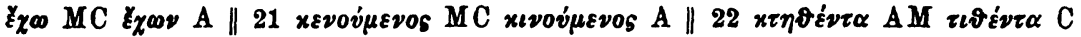




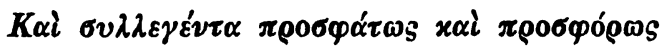

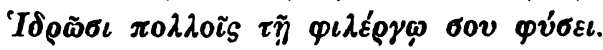

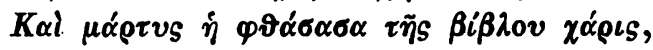

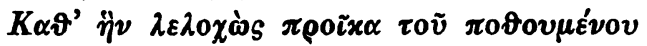

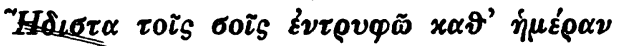

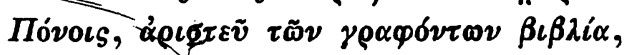

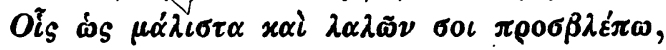

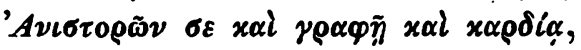

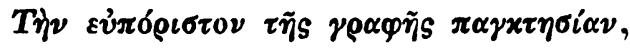

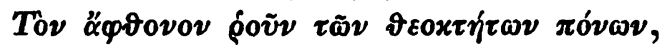

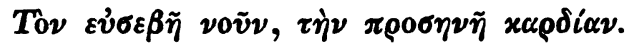

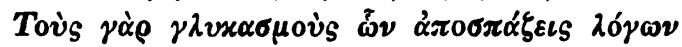

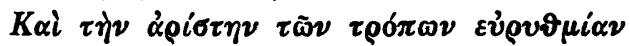

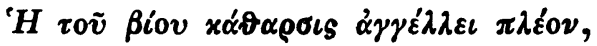

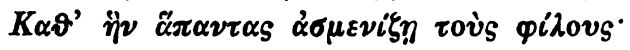

$\Lambda \alpha \lambda \tilde{\omega} \nu \delta \dot{\varepsilon} \pi \alpha \nu \tau \alpha \dot{\pi} \pi \alpha \sigma \iota \nu$ हैv $\pi \alpha \rho \rho \eta \sigma i \alpha$

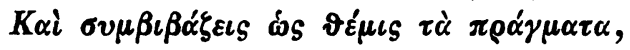

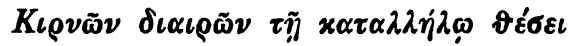

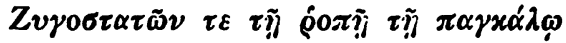

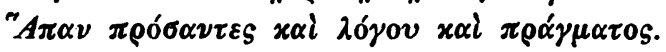

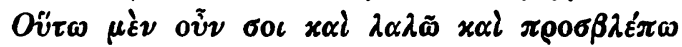

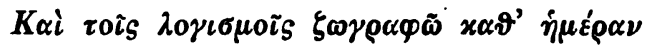

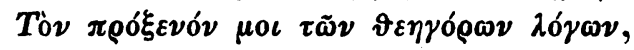

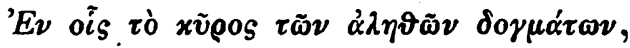

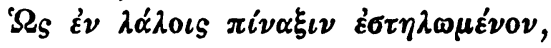

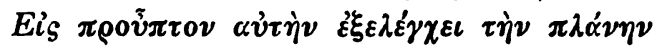

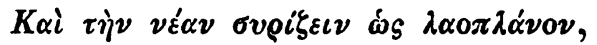

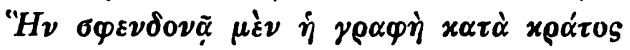

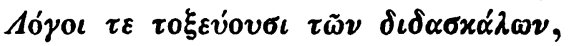

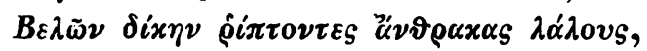

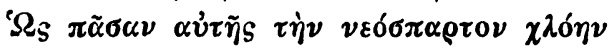

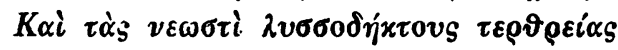

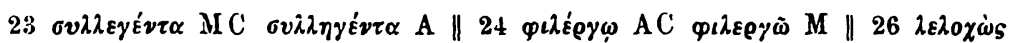
A MC $\lambda \varepsilon \lambda \sigma \gamma \chi \dot{\omega} s$ scrib.? || 29 $\dot{\omega} s$ AC $x \alpha i \mathrm{M} \mid \lambda \alpha \lambda \dot{\omega} \nu \mathrm{MC} x \alpha \lambda \dot{\omega} \nu$ A | $\pi \rho \circ \sigma \beta \lambda \varepsilon \pi \omega \mathrm{AC}$

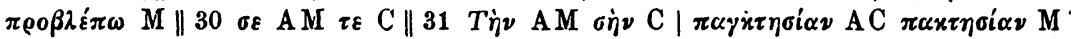
$36 \dot{\alpha} \gamma \gamma \dot{\xi} \lambda \lambda \varepsilon \iota \mathrm{M} \dot{\alpha} \gamma \gamma \xi \hat{\lambda} \varepsilon \iota \mathrm{A} \dot{\alpha} \gamma \gamma \varepsilon \lambda \varepsilon i \mathrm{C}\|38 \Lambda \alpha \lambda \tilde{\omega} \nu \mathrm{AM} \lambda \alpha \lambda \varepsilon i \nu \mathrm{C}\| 39 \sigma v \mu \beta \iota \beta \dot{\alpha} \xi \varepsilon ı \mathrm{~S} \mathrm{MC}$

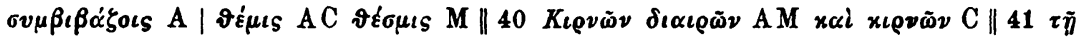

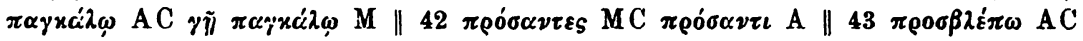

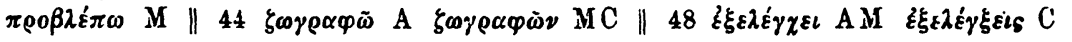

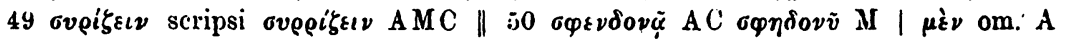

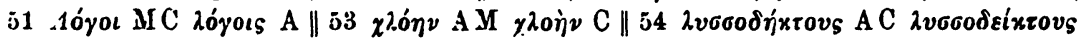

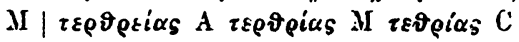




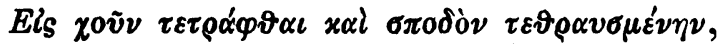

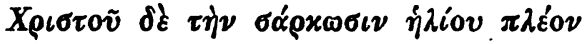

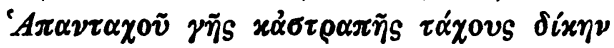

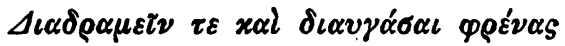

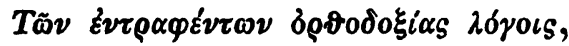

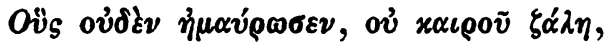

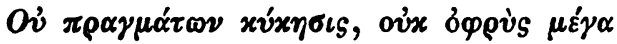

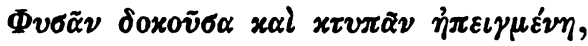

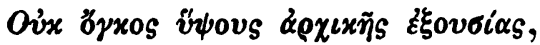

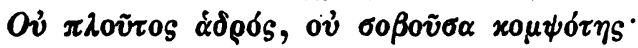

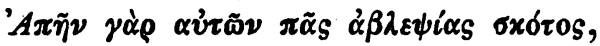

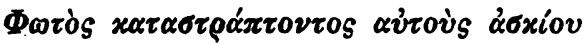

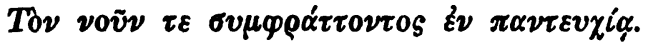

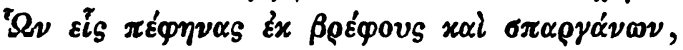

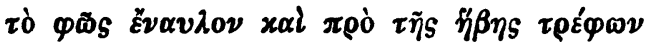

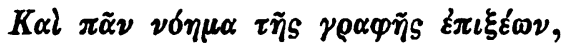

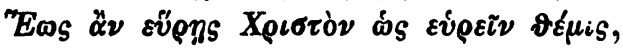

' $\Omega_{S}$ 甲

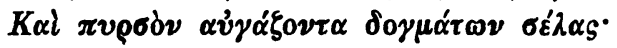

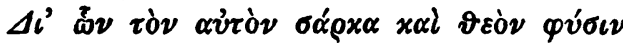

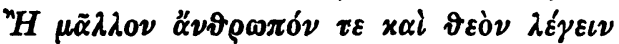

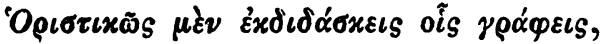

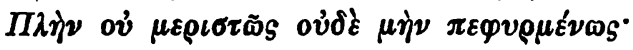

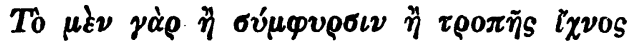

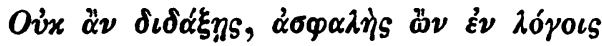

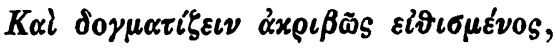

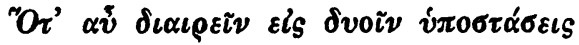

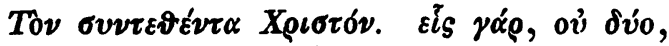

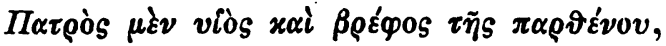

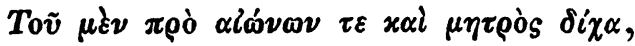

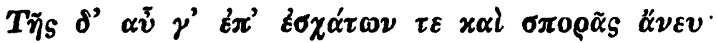

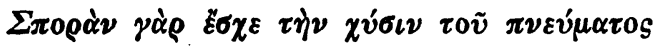

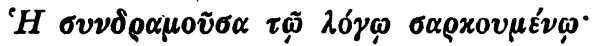

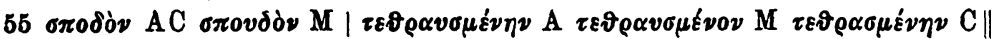

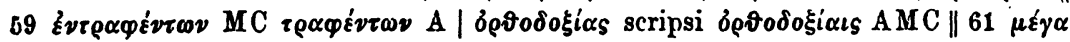

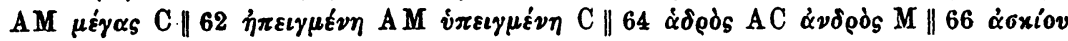

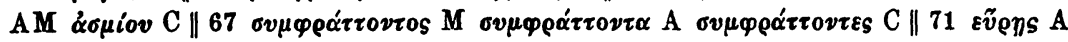

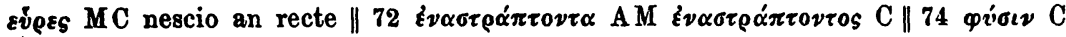

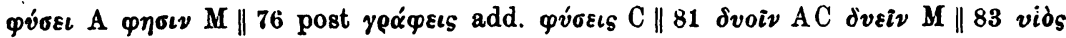

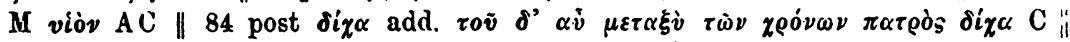

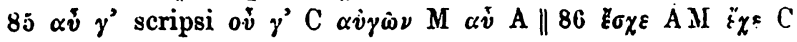




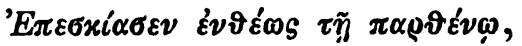

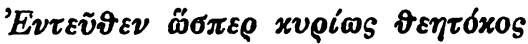

' $\Omega_{S} \alpha \dot{\tau} \tau \dot{\nu} \nu$

90

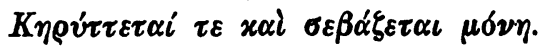

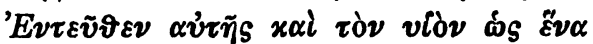

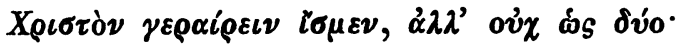

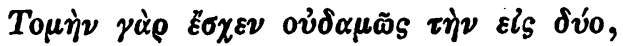

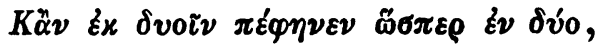

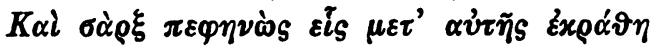

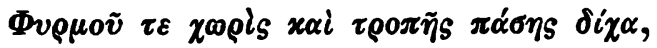

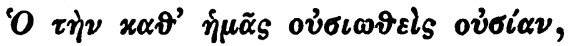

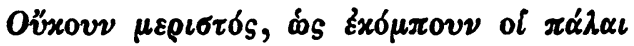

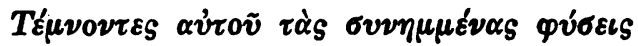

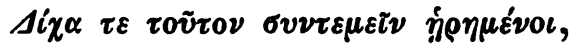

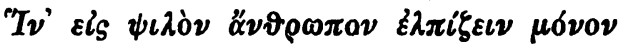

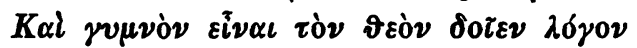

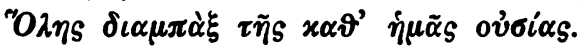

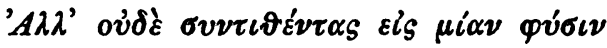

105

$T \grave{\alpha} S \sigma v \nu \delta \rho \alpha \mu 0 v \sigma \alpha \alpha_{S} \varepsilon l_{S} \dot{v} \pi \delta \sigma \tau \alpha \sigma \iota \nu \mu i \alpha \nu$

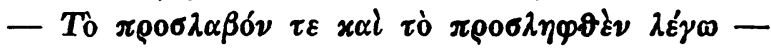

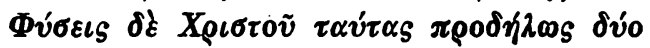

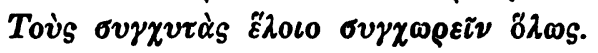

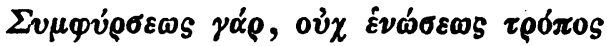

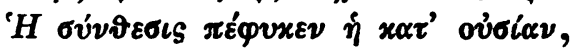

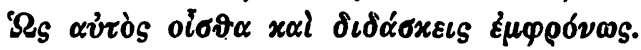

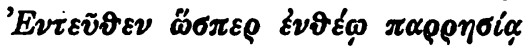

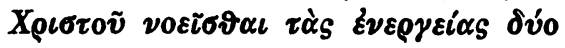

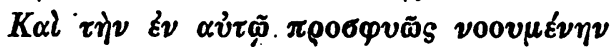

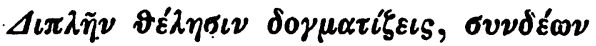

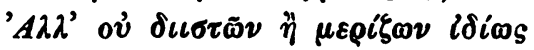

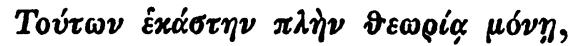

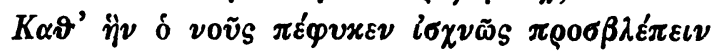

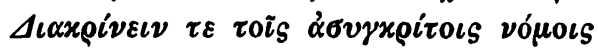

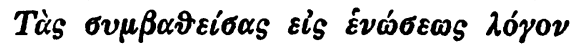

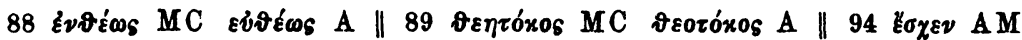

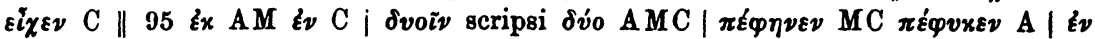

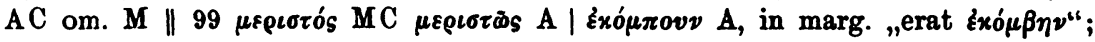

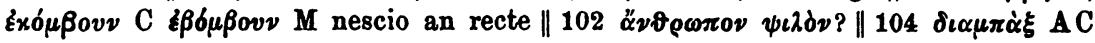

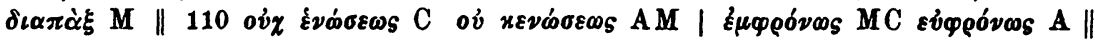

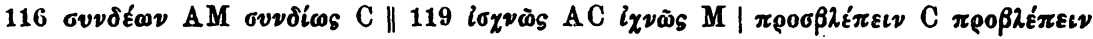
$\mathrm{AM} 甘 121 \sigma v \mu \beta \alpha \vartheta \varepsilon i \sigma \alpha s$ AC $\sigma v \mu \beta \alpha \vartheta \rho \varepsilon i \sigma \alpha s \mathrm{M} \sigma v \mu \beta \alpha \lambda \varepsilon i \sigma \alpha s ?$ 


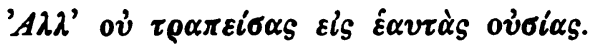

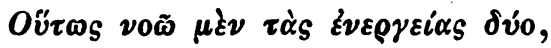

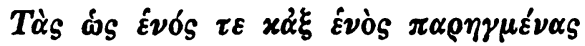

กiov $\tau \varepsilon$ X

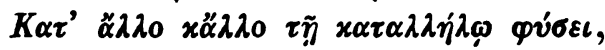

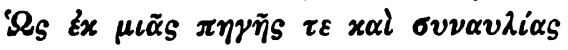

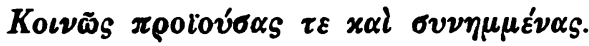

\section{III.}

Andreas hat seine Jamben im ganzen nach den strengen Regeln der antiken Prosodie gebaut. Vor allem ist bei ihm noch keine Rede von der ausschlaggebenden Bedeutung der Silbenzahl, sondern er achtet streng auf Quantität; Nachlässigkeiten sind mir nur wenige aufgefallen, nämlich V. $102 \psi \iota \lambda \dot{o} \nu$ als Jambus, wo durch Umstellung ä $\nu \vartheta \rho \omega \pi 0 \nu$ $\psi \iota \lambda \grave{\nu} \nu$ zu helfen wäre, aber auch dann noch der Vers Schwierigkeiten böte, sodafs wohl ein schlimmerer Fehler im Verse steckt; aufserdem sind V. $108 \pi \rho o ̄ \delta \dot{\lambda} \lambda \omega s$ und V. 117 i $\delta i \omega s$ prosodische Licenzen, gegen die man nicht viel einwenden, wird. Das Gesetz des barytonischen Versausganges ist ein paarmal verletzt worden, V. 19 ๆ

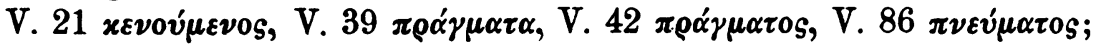
dagegen habe ich keine VerstöIse gegen das Hilbergsche Gesetz gefunden, welches eine accentuierte Endsilbe von der Hephthemimeres ausschliefst, wenn nicht eine Penthemimeres vorhergeht. V. 95 habe

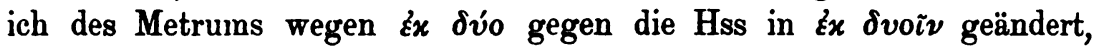
V. $26 \lambda \varepsilon \lambda \bar{\chi} \chi \omega \dot{s}$ dagegen wagte ich nicht durch $\lambda \varepsilon \lambda \sigma \gamma \chi \omega \dot{s}$ zu ersetzen; vgl. übrigens V. $31 \pi \alpha x \tau \eta \sigma i \alpha \nu$ in $M$ statt $\pi \alpha \gamma x \tau \eta \sigma i \alpha \nu$.

Das Versmals des jambischen Trimeters war dem Andreas von Kreta, der mit Virtuosität die Gesetze der rhythmischen Dichtung handhabte, nicht recht geläufig. Er hatte nicht den Mut der späteren Byzantiner, sich von den Regeln der Schule freizumachen; sie waren ihm aber nicht vertraut genug, dafs er sie dem Inhalte hätte unterordnen können. So sind seine Verse keineswegs klar und leicht verständlich geworden; namentlich fehlt es an der logischen Verbindung der Gedanken. Trotzdem ist der Inhalt des Gedichtes nicht mifszuverstehen, es ist ein Bekenntnis des orthodoxen Glaubens gegenüber den Häresien des Nestorianismus, der Monophysiten und der Monotheleten.

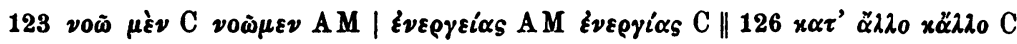

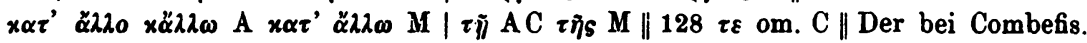

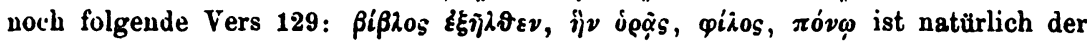
Vermerk eines Schreibers und gehørt nicht zum Gedichte des Andreas; er ist auch in den Hss deutlich davon getrennt. 
Der Adressat des Gedichtes wird in Titel unzweideutig genannt, es ist der Archidiakon und Chartophylax Agathon in Konstantinopel, wie der Abschreiber der Hs A das $\varepsilon v \tau \alpha \tilde{v} \vartheta \alpha$ der Uberschrift richtig interpretierte. Also verfalste Andreas diese Verse zu einer Zeit, da er selbst, in der Hauptstadt weilte.

In Jahre 681 selbst können übrigens diese Verse nicht geschrieben worden sein. Damals war ihr Verfasser noch keinesfalls Erzbischof von Kreta, und Agathon auch noch nicht Archidiakon und Chartophylax. Dieser Mamm ist uns nicht unbekannt, er wird in den Akten des 6. ökunnenischen Konzils erwähnt als einer der unteren Beamten der Kanzlei des Patriarchats. Damals bekleidete ein gewisser Konstan-

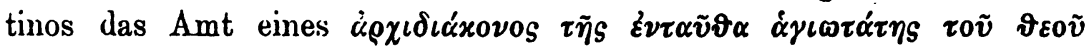

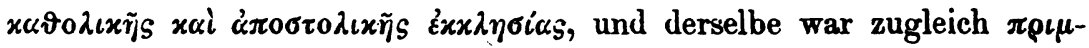

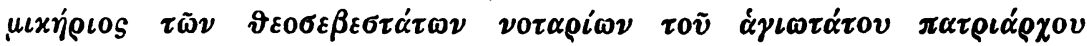
(Harduin a. a. 0 . III col. 1256). Als sein Untergebener fungierte in

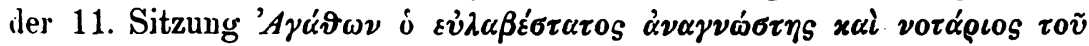

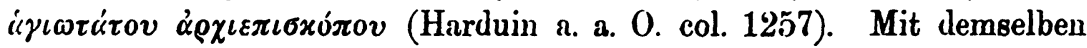
Titel finden wir ihn in dem Protokoll der 12. Sitzung wieder (Harduin ,

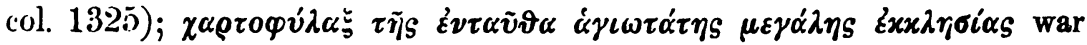
danals ein $\Gamma \varepsilon \dot{\varrho} \varrho \gamma \iota 0$ s (Harduin col. 1324). So werden wir in eine viel spätere Zeit geführt, in der Agathon sowohl wie Andreas zu ihren hohen Würden bereits emporgestiegen waren.

Die Veranlassung des Gedichtes ist klar; es wurde zugleich als Dank einem Buche beigegeben, das Agathon dem Andreas geliehen hatt und das dieser jetzt wieder zurücksandte, nachdem er es sorgfältig abgeschrieben hatte (Überschrift und V. 3). Dafs dieses Buch ein Werk des Agathon selbst war, lehren unwiderleglich die Verse $25 \mathrm{ff}$. Wir wissen auch genau, welches Werk des Archidiakon gemeint war, doch ist zur Erklärung etwas weiter auszuholen. Der Sieg der orthodoxen Kirche schien in den ersten Jahren nach dem 6. Konzil ein allgemeiner und entscheidender zu sein; allmählich aber regte sich der Monotheletismus aufs neue, und der Kaiser Philippikos Bardanes berief eine Synode, welche das 6 . Konzil verurteilte und seine Beschlüsse für null und nichtig erklärte. Kein einziger Bischof der oströmischen Kirche fand den Mut zu widersprechen, der vielgeschmähte Makarios übertraf sie alle an Bekennermut und Überzeugungstreue. Unter den nachgiebigen Kirchenfürsten befand sich auch Andreas von Kreta, wie Theophanes ausdrücklich berichtet. ${ }^{1}$ ) Freilich stellte nach wenigen

1) Ed. de Boor I 362, 21; 382, 17. Vgl. Baronius annales ed. Theiner XI-XII ann. 712.

Byzant. Zeitschrift X 3 u. 4. 
Jahren Anastasios II (713-716) dia Orthodoxie wieder her. Gerade über diesen Umschẉung der Verhältrisse sind wir ziemlich genau unterrichtet durch den Archidiakon Agathon. Philippikos Bardanes hatte die in der kaiserlichen Kanzlei aufbewahrten Akten des 6. Konzils verbrennen lassen. Jetzt wurde Agathon vom Patriarchen Johannes beauftragt, nach den im Patriarchalarchiv aufbewahrten Sitzungsprotokollen einen neuen Bericht auszuarbeiten. Dieser Bericht ist uns erhalten mitsamt einem Epilog des Agathon, der uns über diese Verhältnisse genau unterrichtet, und mit einem von Agathon verfafstęn Briefe des Patriarchen Johannes an den Papst Konstantin ${ }^{1}$ ), in welchen die Orthodoxie der griechischen Kirche feierlich gelobt wird. Es ist nun kein Zufall, dafs mit Ausnahme des späten Sammelcodex A diese 128 Trimeter des Andreas von Kreta in allen Handschriften hinter oder - wie z. B. im Vindob. Hist. gr. 59 - vor dem Berichte über die 6. Synode überliefert sind. Die $\pi \alpha \rho 0 v \sigma \alpha \beta i \beta \lambda o s$, welche der Erzbischof von Agathon erhalten hatte und die er abschrieb und mit den Begleitversen versah, ist also eben jener nicht vor 713 abgefafste Bericht des Agathon über das 6. Konzil. ${ }^{2}$ )

Eigentümlich ist in der Ubberschrift des Gedichtes der Ausdruck

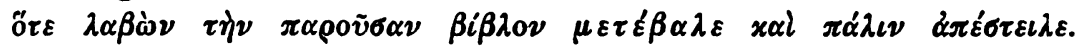
„Accipiens transcripsit ac iterum remisit" übersetzte Combefis, „accepto hoc libro descripsit et rursus remisit" änderte Gallandius. Mir erscheint es sehr zweifelhaft, dafs $\mu \varepsilon \tau \varepsilon \varepsilon \beta \alpha \lambda \varepsilon$ diese Bedeutung haben könnte, um so weniger, als es sich doch nur um eine wörtliche $\mathrm{Ab}$ -

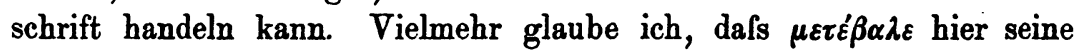
übliche Bedeutung hat „er änderte seine Anschauung", d. h. er bekehrte sich wieder zur Orthodoxie, wie sie nach dem ihm gesandten Werke des Agathon das 6. Konzil festgestellt hatte. 'So ist denn dieses Gedicht mehr als eine poetische Spielerei und mehr als eine Danksagung an einen Freund; es ist das religiöse, in diesem Falle also auch das sehr politische Glaubensbekenntnis des Erzbischofs gewesen, charakteristisch für den Verfasser und bemerkenswert in der Geschichte des Monotheletismus.

München.

Ang. Heisenberg.

1) Ed. Combefis a. a. O. col. $200 \mathrm{ff}$. und ofter.

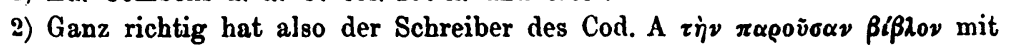

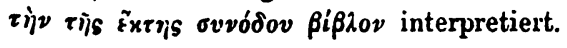

